\title{
One Hundred 30 Dors?
}

\author{
M. M. Hanson and B. Popescu \\ Department of Physics, University of Cincinnati, Cincinnati, OH, USA \\ email: margaret.hanson@uc.edu, popescb@email.uc.edu
}

\begin{abstract}
There are a few ways to estimate the number of massive open clusters expected in the disk of the Milky Way, such as the total star formation rate of the Galaxy, or the open cluster mass function extrapolated to include the entire Galaxy. Surprisingly, they give similar predictions: the Milky Way should contain about 100 clusters as massive as 30 Doradus. Are we seeing them? We look closely at these predictions and compare them to what has been found so far in our Galaxy. We present sophisticated image simulations our group is developing to estimate the selection biases faced by current infrared searches for these massive clusters.
\end{abstract}

Keywords. Galaxy: stellar content - open clusters and associations: general - stars: early-type

\section{Does the Milky Way contain, 'Super Star Clusters'?}

Paul Hodge (1961) was among the first to study very young massive clusters; these are extragalactic clusters of very high mass, but significantly younger than the known globular clusters. The popular term, 'super star cluster', was coined by Sydney van den Bergh (1971). He used it to describe a dozen enormously bright knots of emission seen in the nucleus of M82. However, this new term did not come into popular use until the Hubble Space Telescope era of high-resolution ultra-violet imaging of merging and starbursting galaxies (Holtzman et al. 1992; Whitmore et al. 1993, O'Connell, et al. 1994). Perhaps among the first to use the phrase super star cluster to describe a Milky Way cluster was Serabyn et al. (1999) and Knodlseder (2000), in their studies of the Arches and Cyg OB2 clusters, respectively. Since then, the term has been used to describe other Milky Way clusters, most recently, Westerlund 1. At the time of this contribution, Westerlund 1, with a mass of nearly $10^{5} \mathrm{M}_{\odot}$, is the most massive young cluster known in the entire Local Group (Clark \& Negueruela 2002, Brandner et al. 2008, Negueruela et al. this volume).

Should we have expected to find such massive, young stellar clusters lurking in the inner Milky Way? As surprising as it may at first sound, it is consistent with most observations (and theory!) that our Galaxy should contain numerous young star clusters similar in scale to R136 in 30 Doradus $\left(>10^{4} \mathrm{M}_{\odot}\right)$. It is important to recognize that a mass of just a few $\times 10^{4} \mathrm{M}_{\odot}$ is clearly at the low-mass tail of the 'super star cluster' mass distributions typically studied by extragalactic astronomers (see for example, Whitmore \& Schweizer 1995; Zhang \& Fall 1999; who report on young clusters with $M>10^{6} \mathrm{M}_{\odot}$ ). Nonetheless, the idea of super star clusters being found within the Milky Way is a highly novel and still heavily debated notion.

\section{Predicting the Super Star Cluster Population in the Milky Way}

There are several compelling arguments which provide reasonable and consistent estimates of the number of massive clusters our Milky Way galaxy should be expected to harbor. 


\subsection{The Global Star Formation Rate of Normal Spiral Galaxies}

A Galactic astronomer can become jealous with the full view of the grand design spiral arms and near complete census of star clusters allowed extragalactic astronomers studying face-on galaxies. It is of great interest to know how our Milky Way galaxy would appear among its spiral galaxy brethren. But direct comparisons are hard since our knowledge of the Milky Way's exact properties are so poorly known. We do however have a reasonable estimate of its global star formation rate, 2-5 $\mathrm{M}_{\odot} \mathrm{yr}^{-1}$ (Prantzos \& Aubert 1995; Naab \& Ostriker 2006;). Larsen \& Richtler (2000) have studied young massive clusters found in nearby, normal spiral galaxies. They have shown that among these normal disk starforming spiral galaxies, a useful correlation is found between the galaxies global star formation rate and it's most massive, young cluster. In Figure 1, we show an image taken directly from Weidner et al. (2004, their Figure 3) that graphs this relationship. The rectangles represent the Milky Way star forming 'regions' of Taurus and Orion, while the rectangle at higher mass represents 30 Doradus in the LMC. Based on its global star formation rate, Weidner et al. (2004) argue that the most massive young cluster in the

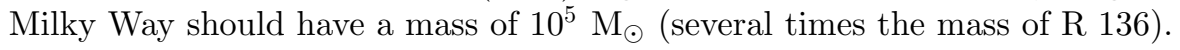

\subsection{Extrapolating the Locally derived Cluster Mass Function}

The mass function of stellar clusters has been measured within the Galaxy, in other galaxies, and in extreme starbursting systems. Extreme starbursting systems, such as the Antennae Galaxies, show clusters of extremely high masses, many over $10^{6} \mathrm{M}_{\odot}$ or even $10^{7} \mathrm{M}_{\odot}$ (Zhang \& Fall 1999) yet, the mass function follows a simple power law, $\delta N / \delta M \propto M^{-2}$. Among normal, non-merging or bursting galaxies, a power-law of slope -2 is also found (Larsen 2002). Within our Galaxy, it was long ago shown by van den Bergh \& Lafontaine (1984, vdBL), that the nearby open clusters follow a power law mass distribution, with slope -2 . Even embedded young clusters measured in the Milky Way

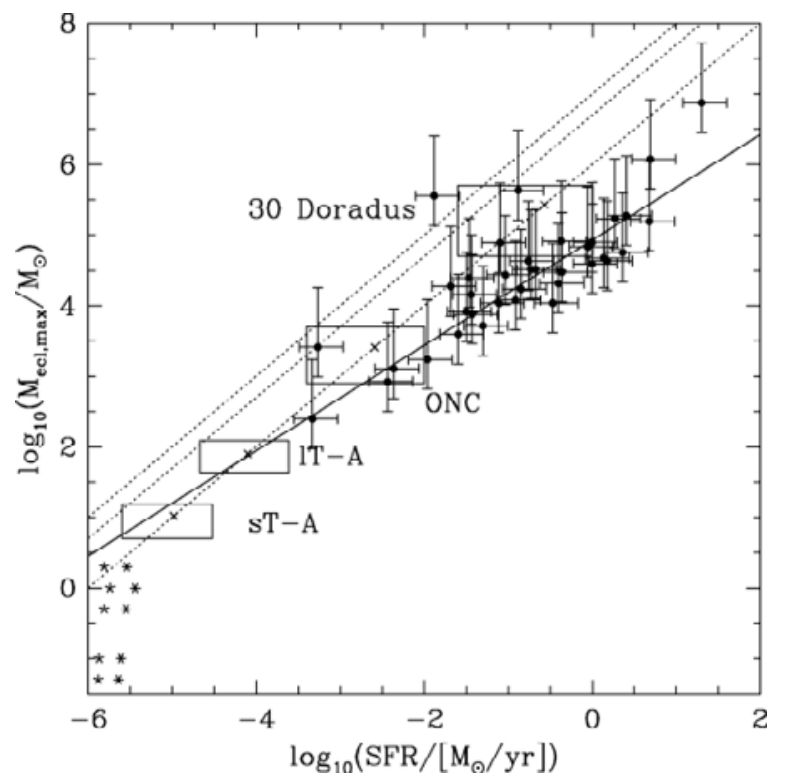

Figure 1. The global star formation rate of normal, disk-star-forming, spiral galaxies verses the most massive young cluster observed in that galaxy. The global star formation rate of the Milky Way is 2-5 $\mathrm{M}_{\odot} \mathrm{yr}^{-1}$, suggesting the most massive cluster present in the Milky Way should have a mass of about $10^{5} \mathrm{M}_{\odot}$. Reproduced from Weidner et al. (2004) with author permission. 
Table 1. Current Census of Massive Young Clusters in the Milky Way ${ }^{1}$

\begin{tabular}{lccccl}
\hline Cluster & l(deg) & Dist $(\mathbf{k p c})$ & Age $(\mathbf{m y r})$ & Mass $\left(\mathbf{M}_{\odot}\right)$ & Mass Reference \\
\hline Westerlund 1 & 339 & 3.5 & $4-5$ & 50,000 & Brandner et al. 2007 \\
RSGC2 & 26 & 5.6 & $15-20$ & 40,000 & Davies et al. 2007 \\
W49a & 43 & 11.8 & $<2 ?$ & $\ldots$ & Homeier \& Alves 2005 \\
RSGC1 & 25 & 5.8 & $10-14$ & 30,000 & Davies et al. 2008 \\
GC Central & 0 & 8 & 6 & 20,000 & Paumard et al. 2006 \\
Arches & 0 & 8 & $2-4$ & 20,000 & Figer et al. 2002 \\
Quintuplet & 0 & 8 & $3-5$ & 20,000 & Figer et al. 1999 \\
NGC 3603 & 291 & 7 & $<2.5$ & 12,500 & Harayama et al. 2008 \\
Westerlund 2 & 284 & 2.8 & 2 & 10,000 & Ascenso et al. 2007 \\
DBS2003 179 & 348 & 8.8 & $3-5$ & 8,000 & Borissova et al. 2008 \\
CL 1806-20 & 10 & 9 & $3-4 ?$ & 3,000 & Bibby et al. 2008 \\
Glimpse 30 & 298 & 7.2 & $4-5$ & 3,000 & Kurtev et al. 2007 \\
\hline
\end{tabular}

${ }^{1}$ Taken mostly from Messineo et al. (this volume)

galaxy show the same power-law slope (Lada \& Lada 2003). It has also been argued on theoretical grounds (Elmegreen \& Efremov 1997, Weidner \& Kroupa 2006) that a power-law mass distribution should be near universal.

Calibrating to the known clusters of the time, vdBL extrapolated the observed luminosity function to include the entire disk of the Galaxy. Such an exercise suggested 100 clusters should exist within the Galaxy with $M_{V}=-11$, about the luminosity of R136. Such a notion seemed 'hard to believe' at the time, and vdBL concluded that the cluster function must 'turn over' above $M_{V}=-8$. This indeed brings up a significant point: is there an upper mass limit for clusters and will this occur globally at the same mass or is it dependent on the galactic system? This question might already have been answered by studies of the luminosity function of extragalactic $\mathrm{H}$ II regions. These observations support the idea that there is a turn over in the luminosity function of H II regions in some galaxies, and it may be a function of the galaxy's Hubble Type (Kennicutt et al. 1989; Oey \& Clarke 1998). McKee \& Williams (1997) claim to see just such a truncation of young massive clusters in our own Galaxy. They estimate the truncation point for making high mass star clusters in the Galaxy is around $2.4 \times 10^{5} \mathrm{M}_{\odot}$ and predict there may be as many as 10 young stellar clusters with mass $10^{5} \mathrm{M}_{\odot}$ presently in the Milky Way (C. McKee, private communication).

\section{What is the census thus far of the Milky Way's super star clusters?}

Beginning with the 2MASS infrared survey (Skrutskie et al. 2006) and the presently ongoing infrared surveys using the Spitzer Space Telescope, numerous groups have invested enormous energy into searching for extinguished young star clusters in the inner Galaxy. Messineo et al. (this volume) provide a current table we will reproduce here (Table 1) with a few additions and updates (Borissova et al. 2008, Bibby et al. 2008). Ages and particularly masses for all of the clusters listed here can differ in the literature, sometimes greatly, based on the method of study. But, we expect the masses to be secure to perhaps a factor of 2. Eight clusters are listed to have reasonably secure masses of at least $10^{4} \mathrm{M}_{\odot}$. That represents barely $10 \%$ of the number we predicted based on the Milky Way's observed properties. Interestingly, of those most massive eight clusters, none are seen on the far side of the Galaxy. Assuming the Milky Way to be well balanced in star formation properties, it safe to assume we must be missing at least half the clusters (those 'eight' on the other side of the Galaxy). But could we be missing still more? 


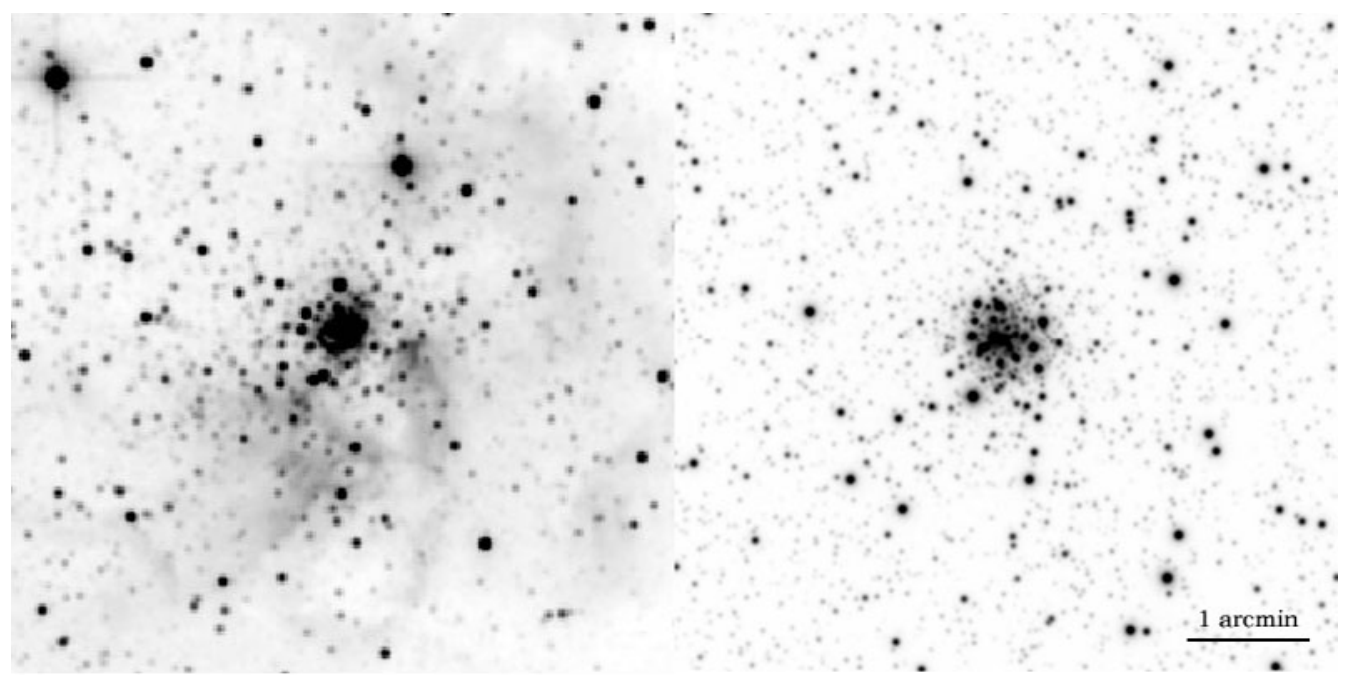

Figure 2. J-Band image of NGC 3603. On the left is the image taken from $2 \mathrm{MASS}^{2}$ Skrutskie (Skrutskie et al. 2006). On the right is our MASSCLEAN simulation, assuming: $\log ($ age $)=6.00$, $M_{\text {total }}=10^{4} M_{\odot}$ and solar metallicity, $A_{V}=4.5 \mathrm{mag}, R_{V}=3.1$, distance $d=6 \mathrm{kpc}$ (distance modulus $13.9 \mathrm{mag}$ ); for the spatial distribution we used $r_{t}=4.4^{\prime}$ and $r_{c}=0.4^{\prime}$ and small segregation rate; Kroupa IMF with $\alpha_{1}=0.3, \alpha_{2}=1.3$ and $\alpha_{3}=2.4$.

\section{Deriving the selection effects of current infrared surveys}

Its difficult to make a quantitative estimate of the clusters that current infrared searches may be systematically missing. Certainly, it is a function of cluster properties (mass, density, age) but also its location in the Galaxy and line of sight extinction. All these characteristics need to be considered in estimating the selection effects of current imaging searches. In order to answer this question, we are developing a sophisticated and rigorous program which accurately simulates images of very massive clusters with a variety of characteristics (age, stellar density, distance, extinction) in the infrared. These will be used with current infrared search algorithms to directly determine the selection effects as a function of cluster characteristics and location in the Galaxy.

We have completed the first stage in our simulation program, called MASSCLEAN (massive cluster Evolution and analysis package, Popescu \& Hanson 2008, in prep.). With a surprisingly small number of parameters we are able to accurately construct the entire evolution of a cluster. The cluster model is built in a modular way. For the mass distribution of the stars in the cluster we use a Kroupa-Salpeter initial mass function [IMF] (Kroupa 2002; Salpeter 1955). The versatility of the package allows us to use a one-, twoor three-power law function (Kroupa-Salpeter type) as the IMF. A truncated IMF is also supported (Oey \& Clarke 2005). The stellar evolution is given by the Geneva Database (Lejeune \& Schaerer 2001). This provides absolute magnitudes for all stars in the cluster in all the needed photometric bands over an enormous range of evolutionary stages (ages). We apply the CCM Model (Cardelli, Clayton \& Mathis 1989) for extinction to each stars photometric band and a distance modulus to produce apparent magnitudes. The spatial distribution of stars within the cluster on the sky is given by the King Model (King 1962). Combining all this, MASSCLEAN can then generate simulated FITS images using SkyMaker (Bertin 2001; Bertin and Fouqué 2007) for a range of masses, ages, distances, extinctions, and stellar density. 
Our MASSCLEAN package has been tested against several real clusters and over a mass range of $10^{3}-10^{6} M_{\odot}$. One thing not included in the simulation is nebulosity (see Fig. 2). However, such morphologies are only significant in the first $1-2$ million years of the cluster's life. Although the package can simulate low mass clusters, we emphasize its use for massive clusters due to the difficulty of such a low mass simulation (lower number of stars to statistically populate the cluster properties). Once we are confident our simulations accurately reproduce massive clusters of known characteristics, we will begin development of completeness tests of current search algorithms to find our simulated clusters within a model of the Milky Way's disk.

\section{Summary}

While infrared searches have greatly increased our ability to study the massive star population of our inner Galaxy, it is not clear just how many massive clusters are still missing from these searches. When considering the Milky Way as like other normal spiral galaxies with similar star formation rates and other such general properties, we may be missing nearly $90 \%$ of these clusters to date. To address this question, we are developing a sophisticated cluster imaging simulation program. The first phase, to develop such a code and test our simulations against known massive clusters, is nearly complete. The next phase is to determine the detectability of such clusters once placed in various locations through out the Galactic disk. It is our hope that we can provide estimates of the completeness of current surveys as a function of cluster properties (distance, extinction, age, core density, etc). This should allow us to derive the biases inherent in current cluster search methods that rely on infrared imaging surveys. We suspect clusters that currently exist in certain age ranges (such as when the red supergiant phase is just starting, 4-5 million years old) and clusters with rather low stellar densities are being missed by the current searches, even if they have very high cluster mass. This will be particularly true if a cluster is behind very high extinction or is at a great distance. Finally, our study of current search algorithms will not only help us better understand the search biases, it may allow us to design a search algorithm which is better tailored to find a higher percent of the massive young clusters presently existing in the inner Milky Way.

\section{References}

Ascenso, J., Alves, J., Vicente, S., \& Lago, M. T. V. T. 2007, A\&\&A, 476, 199

Brandner, W., Clark, J. S., Stolte, A. et al. 2008, A\&A, 478, 137

Bibby, J. L., Crowther P. A., Furness, J. P., \& Clark, J. S. 2008, MNRAS, 386, 23

Bertin, E. 2001, SKYMAKER, http://terapix.iap.fr/cplt/oldSite/soft/skymaker/

Bertin, E. \& Fouqué, P. 2007, SkyMaker, http://terapix.iap.fr/rubrique.php?id_rubrique $=221$

Borissova, J., Ivonov, V., \& Hanson, M. M. 2008, A\& $A$, accepted

Cardelli, J. A., Clayton, G. C., \& Mathis, J. S. 1989, ApJ, 345, 245

Clark, J. S. \& Negueruela, I. 2002, A\&AA, 396, L25

Davies, B. Figer D. F., Kudritzki R.-P. et al. 2007, ApJ, 671, 781

Davies, B., Figer, D. F., Law C. J. et al. 2008, ApJ 676, 1016

Elmegreen, B. G. \& Efremov, Y. N. 1997, ApJ, 480, 235

Figer, D. F., McLean, I. S. \& Morris, M. 1999, ApJ, 514, 202

Figer, D. F. Najarro, F., Gilmore, D. et al. 2002, ApJ, 581, 258

Harayama, Y., Eisenhauer, F., Martins, F. 2008, ApJ, 675, 1319

Hodge, P. W. 1961, ApJ, 133, 413

Holtzman, J. A., Faber S. M., Shaya, E. J. et al. 1992, AJ, 103, 691

Homeier, N. L. \& Alves, J. 2005, A\&A, 430, 481

Kennicutt, R. C., Edgar, B. K., Hodge, P. 1989, ApJ, 337, 791 
King, I. 1962, $A J, 67,471$

Kroupa, P. 2002, Sci, 295, 82

Kurtev, R., Borissova, J., Georgiev, L., et al. 2007, A\&GA, 475, 209

Lada, C. J. Lada, E. A. 2003, ARA $\mathscr{S} A$ 41, 57

Larsen, S. S. \& Richtler, T. 2000, $A \mathscr{E} A, 354,836$

Larsen, S. S. 2002, $A J, 354,836$

Lejeune, T. \& Schaerer, D. 2001, A\&A, 366, 538

McKee, C. F. \& Williams, J. P. 1997, ApJ, 476, 144

Naab, T. \& Ostriker, J. P. 2006, MNRAS, 366, 899

O'Connell, R. W., Gallagher, J. S., Hunter, D. A. 1994, ApJ, 433, 65

Oey, M. S. \& Clarke, C. J. 1998, AJ, 115, 1543

Oey, M. S. \& Clarke, C. J. 2005, ApJ, 620, L43

Paumard, T. Genzel, R., Martins, F. et al. 2006, ApJ, 643, 1011

Prantzos, N. \& Aubert, O. 1995, A\&A, 302, 69

Salpeter, E. E. 1955, ApJ, 121, 161S

Serabyn, E., Shupe, D. \& Figer, D. F. 1998, Nature, 394, 448

Skrutskie, M. F., Cutri, R. M., Stiening, R., et al., 2006, AJ, 131, 1163

van den Bergh, S 1971, A\& A, 12, 474

van den Bergh, S, Lafontaine, A. 1984, AJ, 89, 1822

Weidner, C., Kroupa, P. \& Larsen, S. S. 2004, MNRAS 350, 1503

Weidner, C., Kroupa, P. 2006, MNRAS 365, 1333

Whitmore, B. C. Schweizer, F., Leitherer, C. et al. 1993, AJ, 106, 1354

Whitmore, B. C. \& Schweizer, F. 1995, AJ, 109, 960

Zhang, Q, Fall, M. 1999, ApJL, 527, 81

\section{Discussion}

OEY: Just a comment that unlike the local stellar mass function, which seems to show a universal upper mass cutoff, clusters do not have a universal upper mass cutoff. Oey \& Clarke 1998 showed that the form of the H II region liminosity function demonstrates that the cluster upper-mass cutoff varies with Hubble type and the Milky Way is an Sb Galaxy. So you would not necessarily expect to extrapolate the power low mass function to infinity. The same has been shown by Kennicutt in the 1980's.

LEITHERER: The plot showing the location of the Galaxy relative to other objects has a misleading abscissa. One should not plot star-formation rate but the specific star formation rate, i.e. normalized to unit surface area. In your units, the Galaxy and the starburst prototype M82 would have nearly the same x-location because their total star formation rates are similar. Yet the specific rates differ by a factor of 100 because M82 is a dwarf galaxy. Would you use M82 as a guide for the Milky Way because there are hundreds of luminous clusters in M82?

HAnson: The plot I showed (Figure 1 in this proceedings) includes only normal, noninteracting spiral galaxies with normal disk star formation similar to what we would expect for the Milky Way. 